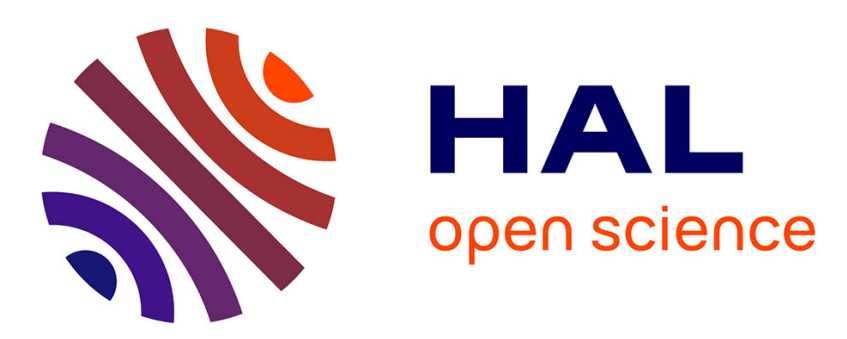

\title{
Using magnetic disturbances to improve IMU-based position estimation
}

\author{
David Vissière, Alain Pierre Martin, Nicolas Petit
}

\section{To cite this version:}

David Vissière, Alain Pierre Martin, Nicolas Petit. Using magnetic disturbances to improve IMUbased position estimation. European Control Conference 2007, Jul 2007, Kos, Greece. pp.2853-2858. hal-00563412

HAL Id: hal-00563412

https://hal-mines-paristech.archives-ouvertes.fr/hal-00563412

Submitted on 4 Feb 2011

HAL is a multi-disciplinary open access archive for the deposit and dissemination of scientific research documents, whether they are published or not. The documents may come from teaching and research institutions in France or abroad, or from public or private research centers.
L'archive ouverte pluridisciplinaire HAL, est destinée au dépôt et à la diffusion de documents scientifiques de niveau recherche, publiés ou non, émanant des établissements d'enseignement et de recherche français ou étrangers, des laboratoires publics ou privés. 


\title{
Using magnetic disturbances to improve IMU-based position estimation
}

\author{
David Vissière, Alain Martin, Nicolas Petit
}

\begin{abstract}
We address the problem of position estimation for a rigid body using an inertial measurement unit (IMU). In this paper, we present a Kalman filtering technique which takes advantage of the magnetic disturbances usually observed indoors. This is an important topic for military operations in urban areas where GPS is often unavailable. This approach yields significant improvements in estimation accuracy. We illustrate our technique with several experimental results obtained with a low cost IMU.
\end{abstract}

\section{INTRODUCTION}

Numerous military and civilian control applications require high accuracy position, speed and attitude estimations of a solid body. Examples range from Unmanned Air Vehicles (UAV), Unmanned Ground Vehicles (UGV), full-sized submarines, sub-sea civil engineering positioning devices [11], to name a few. A widely considered solution is to use embedded Inertial Measurement Units (IMU). Accelerometers, gyroscopes (and possibly magnetometers) signals can be used to derive position information through a double integration process [5], [4]. Because of sensors drifts, this approach requires very high precision IMUs such as those found in certain full-sized aircrafts, military submarines, and missiles. When cost, space, and weight constraints become stringent, other solutions need to be used. Two prime examples are sensors fusion on ground vehicles equipped with odometers and GPS (for outdoor and non military critical applications as considered in [8]), and computer vision through cameras for indoor robotics. It is interesting to get an idea of prices and obtained performance. Typically, a ground navigator based on IMU measurements used for petroleum research costs more than 20,000 USD, while its position estimates drift by 3 Nautic miles per hour (when the navigation system is used only during maneuvers and manually forced to a zero update around steady points, the drift in position can be reduced to $100 \mathrm{~m}$ for a 3 Nautic miles trip). A recent trend has been to heavily rely on the well known Global Positioning system (GPS) technology. In facts, this technology is very appealing for low weight, low cost, and low size applications (see e.g. [7]). Yet, it suffers from major drawbacks. It has a limited availability (especially in the context of military operations), its accuracy is (roughly speaking) of $10 \mathrm{~m}$ of error, and the GPS signal is quite weak.

D. Vissière (corresponding author) is "Ingénieur de l'Armement" in the Délégation Générale pour l'Armement (DGA) of the French Department of Defense. He is also a PhD candidate in Mathematics and Control, Centre Automatique et Systèmes, École des Mines de Paris, 60, bd St Michel, 75272 Paris, France david.vissiere@dga.defense.gouv.fr

A. Martin is "Ingénieur" in the Délégation Générale pour l'Armement (DGA) of the French Department of Defense.

N. Petit is with the Centre Automatique et Systèmes, École des Mines de Paris, 60, bd St Michel, 75272 Paris, France

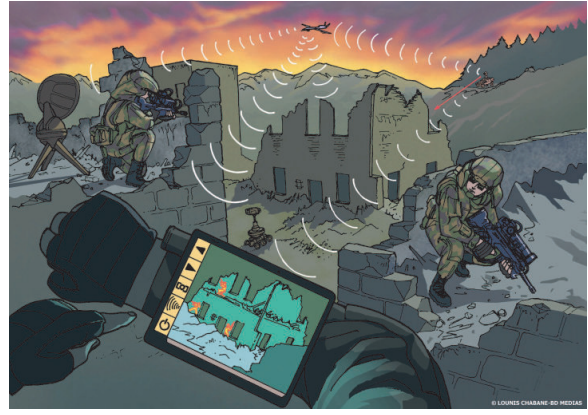

Fig. 1. A typical platoon of soldiers in action as envisioned in the BOA projet.(CL.Chabane-BD Médias for Délégation Générale pour l'Armement (DGA). A team leader keeps track of his soldiers thanks to real-time position information reported on his arm portable display.

GPS is very poorly useable between buildings or in forests. Very importantly, it is not available indoors. Alternative solutions are under development. Most of them rely on computer vision using cameras or lasers (see e.g. [13]), e.g to match partial information from maps or to progressively construct a map of the neighborhood.

Yet, cameras and other optical devices are often to be discarded. In numerous missions (e.g. soldiers or policemen taking control of a building or firemen rescuing people in smoky rooms), lighting conditions, smoke, or high temperature totally prevent camera from being used. The recent progress in very low cost (less than 1,500 USD), low weight (less than $100 \mathrm{~g}$ ) and low size (less than $3 \mathrm{~cm}^{2}$ ) IMUs have spurred a broad interest in the development of IMU-based positioning technologies. These Micro-Electro-Mechanical Systems (MEMS) IMUs appear to have quickly increasing capabilities. Several manufacturers are announcing new models under 5,000 USD capable of less than $20 \mathrm{deg} / \mathrm{hr}$.

So far, there does not exists any reported experiment proposing to estimate the position from such a low cost IMU. In the literature, these IMUs are only used for attitude estimation (see e.g. [3], [9] or [2] for an application to the control of mini-UAVs in closed loop). Some tentative work (using higher-end IMUs) address the problem of velocities estimation. In these cases, the speed information is obtained from a GPS receiver using the Doppler effect (see [5] for details on the quality of the obtained measurements information) or from odometers (in the case of ground vehicles). For ground vehicles, position is usually primarily derived from GPS data complemented with higher frequency measurements from IMU and magnetometer (see e.g. [12]). Our focus in on indoor missions involving humans. It is desired to remotely estimate their positions. During preliminary tests, 


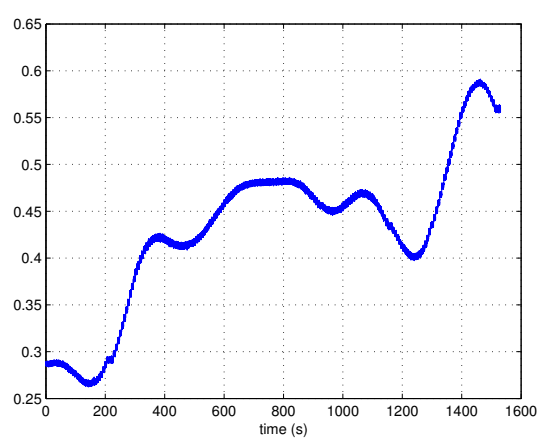

Fig. 2. Variation of the magnetic field norm during a $2.4 \mathrm{~m}$ horizontal displacement inside a business building office.

it quickly appeared to us that, given a poorly known models of the dynamics, it is impossible to get a position error below $50 \mathrm{~m}$ after a few minutes of experiments from a low cost IMU (e.g. a 3DMGX1 from Microstrain $\mathbb{R}$ ). High-end IMUs are usually much too heavy for human-oriented applications. Further, while the GPS signal is unavailable indoor, experimental measurements have shown that the magnetic field in a typical business building is strongly disturbed (by the building structure, electrical equipments, computers, and cell-phones among others). For sake of illustration, we report the variations of the magnetic field norm inside a business building office in Figure 2. Consequently, classic estimation algorithms based on heading measurements are very inefficient in such a context.

Our claim is that these disturbances (which are assumed to be constant) can actually be used to improve the position estimation. Our work is related to the approach advocated in [6] for gravimetry aided navigation. Very importantly, our approach does not rely on any a-priori magnetic map. It simply uses Maxwell's equation. In words, we note that, in a disturbed magnetic field, it is possible to determine when a solid body equipped with a magnetometer is moving. If it moves, then the sensed magnetic field must change according to Maxwell's law. If the magnetic measurements do not change significantly, then the solid body is not moving. This permits to rule out velocity drifts in our estimation. Eventually, this improves the position information obtained by integrating the velocity estimate. Experimental results we present on a preliminary simple testbed, using a very low cost inertial unit lead us to believe it is possible to estimate the position of a man bearing a low cost IMU who is investing a building. This objective fits in the network centric warfare (as defined in [10]) context "Bulle Opérationnelle Aéroterrestre" (BOA), led by the Délégation Générale pour l'Armement (DGA) for the French Department of Defense. A typical mission in the BOA environment is depicted in Figure 1.

The article is organized as follows. In Section I, we define the position estimation problem. Required notations are presented. In Section II, we expose our use of magnetic disturbances. In particular, we focus on eliminating bias in velocity estimates. In Section III, we present experimental results and give implementation details. In Section IV, we conclude and suggest several directions of improvement.

\section{PRoblem STATEMENT}

In this paper, we do not describe in details the dynamics sensed by the IMU. Our primary goal is to stress the information from the magnetic sensor that can be exploited. When actually implementing the estimation filter, we often include numerous terms in the dynamics model for sake of accuracy, but very good estimates can be recovered from the basic equations we present here.

\section{A. Coordinate frames, system of equations, notations}

We consider the motion of an IMU (viewed as a material point) located at the center of gravity of a moving body we wish to estimate the position of. The system can simultaneously rotate and translate. It has six degrees of freedom. A body reference fixed frame with origin at the center of gravity of the IMU can be considered. In that case, the $\mathrm{x}, \mathrm{y}$ and $z$ axis are the IMU axis (i.e. are consistent with the inner sensors orientations). In the following, subscript ${ }_{b}$ refers to this body frame.

As inertial reference frame, we consider the NED frame: North-East-Down, the $X$ axis is tangent to the geoid and is pointing to the north, the $\mathrm{Z}$ axis is pointing to the center of the earth, and the $\mathrm{Y}$ axis is tangent to the geoid and is pointing to the East. Subscript ${ }_{i}$ refers to this inertial frame.

The IMU delivers a 9 parameters vector $Y=$ $\left[\begin{array}{lll}Y_{V} & Y_{\Omega} & Y_{M}\end{array}\right]^{T}$ obtained from a 3-axis accelerometer, a 3 -axis gyros and a 3-axis magnetometer. Measurements are noisy and biased. Classically, we consider that the accelerometer signal $Y_{V}$ has a bias $B_{V}$ (independently on each axis) and suffers from additive white noise $\mu_{v}$, that both the magnetometer signal $Y_{M}$ and the gyros signal $Y_{\Omega}$ have additive white noises $\mu_{M}$ and $\mu_{\Omega}$, respectively. Finally, there is a drift $B_{\Omega}$ on $Y_{\Omega}$. It is possible to consider unknown scale factors to increase filtering accuracy, but these are not necessary in a first approach. We denote by $B_{V}$ the drift off the accelerometer and by $B_{\Omega}$ the drift off the gyros. Noting $F$ the external forces (other than gravitational) acting on the IMU, and $R$ the rotation matrix from the inertial frame to the body frame, we can write the measurement equations

$$
\left.\begin{array}{rl}
Y_{V} & =F-R \vec{g}+B_{V}+\mu_{V} \\
Y_{\Omega} & =\Omega+B_{\Omega}+\mu_{\Omega} \\
Y_{M} & =M+\mu_{M}
\end{array}\right\}
$$

where $\vec{g}$ stands for the gravity, and $M$ is the magnetic field in the body frame. For the bias vector $B=\left[\begin{array}{ll}B_{V} & B_{\Omega}\end{array}\right]^{T}$, several models can be considered depending on accuracy requirements. A second order damped oscillator driven by a white noise is a good choice. Classically, in filter equations, bias will be added in an extended state.

From a dynamical system point of view, the state of the rigid body is described by the 12 following independent variables

- $X=\left[\begin{array}{lll}x & y & z\end{array}\right]^{T}$ is the position of the center of gravity of the IMU in the inertial frame 
- $V=\left[\begin{array}{lll}u & v & w\end{array}\right]^{T}$ is the vector velocity of the center of gravity of the IMU in the body frame

- $Q=\left[\begin{array}{lll}\phi & \theta & \psi\end{array}\right]^{T}$ The Euler angles, i.e. the angles between the inertial frame and the body.

- $\Omega=\left[\begin{array}{lll}p & q & r\end{array}\right]^{T}$ it the angular rate of turn in the body frame

The input vector of the dynamics are the forces $F=$ $\left[\begin{array}{lll}F_{u} & F_{v} & F_{w}\end{array}\right]^{T}$ and torques $\Gamma=\left[\begin{array}{lll}\Gamma_{u} & \Gamma_{v} & \Gamma_{w}\end{array}\right]^{T}$. We call $R$ the rotation matrix between the inertial and the body reference.

Remark: To avoid the well known singularities when angles cross $\frac{\pi}{2}$, quaternions can be used to represent the Euler angles. For sake of simplicity, we do not present quaternions equations but they certainly are handy in this situation.

\section{B. Equations of motion}

The matrix of inertia of the system is unknown. It is approximated by the identity matrix. Models for the unknown forces $F$ and torques $\Gamma$ may be chosen. A basic choice to model them as the output of a first order stable system driven by white noises. Indirectly, the variance of the white noise is used to specify the manoeuvring capabilities of our system.

$$
\begin{gathered}
\dot{F}=-\frac{F}{\tau_{F}}+\nu_{F} \\
\dot{\Gamma}=-\frac{\Gamma}{\tau_{\Gamma}}+\nu_{\Gamma}
\end{gathered}
$$

where $\tau_{F}, \tau_{\Gamma}$ are positive constant. In summary, using the matrix $R$ defined in equation (2), we get the following system dynamics

$$
\left.\begin{array}{rl}
\dot{X} & =R^{T} V \\
\dot{V} & =-\Omega \times V+F \\
\dot{Q} & =G(\Omega, Q) \\
\dot{\Omega} & =\Gamma
\end{array}\right\}
$$

with

$$
G(\Omega, Q)=\left[\begin{array}{c}
p+(q \sin (\phi)+r \cos (\phi)) \tan (\theta) \\
q \cos (\phi)-r \sin (\phi) \\
(q \sin (\phi)+r \cos (\phi)) \cos (\theta)^{-1}
\end{array}\right]
$$

\section{USING MAGNETIC FIELD DISTURBANCES TO INCREASE OBSERVABILITY}

The measurements obtained from the IMU are expressed in the body coordinates frame and are related to a vector in the inertial frame by the matrix $R$ through the relation

$$
M=R M_{i}
$$

The usual way to take the magnetic measurements into account is to consider it gives a direct reading on the attitude, namely a direct measure of magnetic heading vector. This is a quite valid assumption except when there are magnetic disturbances. This approach gives very good results, provided magnetic disturbances are negligible. Yet, as can be seen in Figure 2 and Figure 3, these disturbances are not negligible indoor, e.g. in typical business offices or houses. One can notice the important variations of magnetic heading when

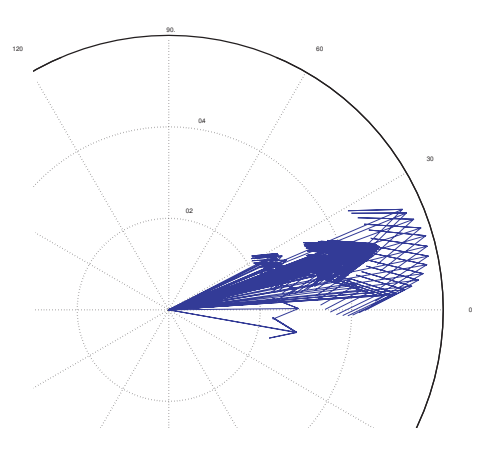

Fig. 3. Variation of (projected) magnetic field during during a $2.4 \mathrm{~m}$ horizontal displacement inside a business building office. Heading information is strongly altered.

making a simple (slow) $2.4 \mathrm{~m}$ horizontal displacement in such a environment, and also remark the large variations of the magnetic field norm during the same horizontal displacement.

Using the inertial frame, we use Maxwell's equations [1] to derive the following three properties

- The magnetic field is stationary. According to Faraday's law of induction in the absence of electrical sources $\frac{\partial M_{i}}{\partial t}=0$. In other words, the magnetic field is a function of the position only. We note it $M_{i}(X)$.

- The magnetic field is a potential field. According to Ampère's law, in the absence of electric and magnetic sources, $\operatorname{curl}\left(M_{i}\right)=0$. Therefore, there exists a scalar function $h(X)$ such that $M_{i}=\nabla h$.

- The divergence of the magnetic field is zero: $\operatorname{div}\left(M_{i}\right)=$ 0 . Thanks to the previous property, this implies $\Delta h=$ $h_{x x}+h_{y y}+h_{z z}=0$

In the body frame, one can differentiate (5) to get the following differential equation thanks to a chain rule

$$
\dot{M}=-\Omega \times M+R \nabla^{2} h R^{T} V
$$

To estimate the gradient of $M$, we perform an estimation in the inertial frame. We extend the state by adding the magnetic field $M$ and the independent gradients $H$. Due to the three properties presented above, there are only five independent gradients to look for. These are, in the inertial frame $H \triangleq$ $\left[\begin{array}{lllll}h_{x x} & h_{x y} & h_{x z} & h_{y z} & h_{z z}\end{array}\right]^{T} \in \mathbb{R}^{5}$.

A first approach (given for its tutorial value) to this estimation problem can be to assume that the gradient of the magnetic field satisfy some first order dynamics driven by white noises $\nu_{H}$. In other words, we can assume that

$$
\dot{H}=-\frac{H}{\tau_{H}}+\nu_{H}
$$

where $\tau_{H}$ is a positive constant. In practice, this model is not really sufficient to capture the richness of the magnetic field gradients dynamics. Typical (from experiment) variations of one such gradient is reported in Figure 4. Depicted slopes suggest second derivatives are not neglectable. For sake of 


$$
R=\left[\begin{array}{ccc}
\cos (\psi) \cos (\theta) & \sin (\psi) \cos (\theta) & -\sin (\theta) \\
-\cos (\phi) \sin (\psi)+\sin (\phi) \sin (\theta) \cos (\psi) & \cos (\phi) \cos (\psi)+\sin (\phi) \sin (\psi) \sin (\theta) & \cos (\theta) \sin (\phi) \\
\sin (\phi) \sin (\psi)+\cos (\phi) \sin (\theta) \cos (\psi) & -\sin (\phi) \cos (\psi)+\cos (\phi) \sin (\psi) \sin (\theta) & \cos (\theta) \cos (\phi)
\end{array}\right]
$$

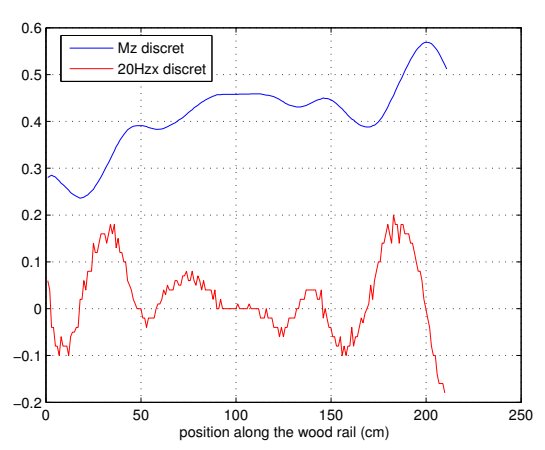

Fig. 4. Histories of $h_{z x}$ the partial derivative of the $\mathrm{z}$ component of the magnetic field in the $\mathrm{x}$ inertial direction during a $2.1 \mathrm{~m}$ move at constant speed along a wood (therefore non-magnetic) rail.

performance, it is recommended to use sophisticated higher order dynamics instead of (7) (angles, and velocities estimate can actually be used in the definition of this dynamics). Finally, with this approach, the extended state we consider has 20 scalar variables. We will use an extended Kalman filter to estimate it. Equation (6) plays a key role in this observation problem. It is the only one giving absolute information on $V$.

\section{FILTER DESIGN AND EXPERIMENTAL RESULTS}

\section{A. Filter design}

The state of our filter is composed of the 12 (independent) configuration states for our 6 DOF IMU, 6 states used to model the torques and forces (with can be optionally complemented by 6 extra state to complete model the dynamic on $F$ and $\Gamma$ with second order dynamics for example), 6 states to model the sensor error (which can become 12 with a second order modelling approach), 3 to model the magnetic field $M, 5$ for its independent derivatives $H$ ( 7 more can be considered using second order models). The state vector used to obtained the experimental results presented in this paper is composed of 38 variables. The filter equations are presented below. In implementation, the covariance matrices can be initialized with values consistent with the ranges of dynamics under consideration (accelerations, speeds...) of our system. The filter updates are synchronized with the $75 \mathrm{~Hz}$ IMU measurements. Classically, discrete update equations are considered. A special attention is paid to maintain the covariance matrices positive. Note $P_{p}$ the $38 \times 38$ covariance matrix of the state used for prediction, $P_{e}$ the $38 \times 38$ covariance matrix of the state used for estimation, $Q$ the $38 \times 38$ covariance matrix used in the noise dynamics, $R$ the $9 \times 9$ covariance matrix considered in the sensor noise definition, $X_{p}$ the 38 dimensional predicted state, $X_{e}$ the 38 dimensional estimated state, $A$ the $38 \times 38$ matrix of the system obtained by linearizing dynamics (4)-(6)-(7), $C$ the $9 \times 38$ matrix obtained by linearizing the measurement equation (1), $\dot{X}=F(X)$, and $T$ the sample time (between measurements updates). We first do a prediction from time $k$ to $k+1$, obtaining $X_{p}$ and $P_{p}$, and then estimate the state through the measurements to obtain $X_{e}$ and $P_{e}$. The updates are computed as follows

$$
\begin{aligned}
X_{p}= & X_{e}+F\left(X_{e}, U\right) T \\
P_{p}= & (I+A T) P_{e}(I+A T)^{T}+Q T+\left(A Q+Q A^{T}\right) \frac{T^{2}}{2} \\
& +A Q A^{T} \frac{T^{3}}{3} \\
Y_{p}= & {\left[F-R \vec{g}+B_{V} ; \Omega+B_{\Omega} ; M\right]^{T} } \\
K= & P_{p} C^{T}\left(R+C P_{p} C^{T}\right)^{-} 1 \\
X_{e}= & X_{p}+K\left(Y-Y_{p}\right) \\
P_{e}= & (I-K C) P_{p}(I-K C)^{T}+K R K^{T}
\end{aligned}
$$

\section{B. Experimental testbed}

Our experimental testbed is designed to illustrate the relevance of exploiting the potential field nature of the magnetic field observed in usual buildings. So far, this (simple) testbed only permits one dimensional movements in a vertical plane. The magnetic field is unknown and has 3-dimensional variation. This enables us to precisely measure displacements.

An inertial measurement unit is fixed on a trolley which can roll along a $2.5 \mathrm{~m}$ wood rail. No a priori information about this motion was taken into account. We use equally valued parameters for the $x$ and $z$ axis in the covariance

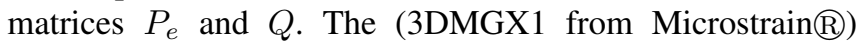
IMU provides measurements at a $75 \mathrm{~Hz}$ rate. The experiment has been used in different rooms in our building. Observed results are very similar.

Tests are conducted as follows. We push (in no particular way) the trolley along the rail from one displacement mark to the next one. This way, we do 3 go-stop-back and then let the IMU to rest. First, we place our test bed outdoor in a non magnetic-perturbed area. It is easily verified that our filter gives similar results wether the gradient of the magnetic field was used or not (i.e. omitting equations (6) and (7) or not). Then, we move our test bed inside a building. For sake of illustration, we also try another experiment by using a 15 degrees inclination for the rail. There is no noticeable difference in the observation accuracy.

Finally, it is important to mention that when using our test bed, we restrict ourselves to a 2-D problem by zeroing the velocity along the $\mathrm{y}$-axis by assuming $\dot{y}=v=0$ and $p=r=0$. A complete three-dimensional experiment is currently in development. 


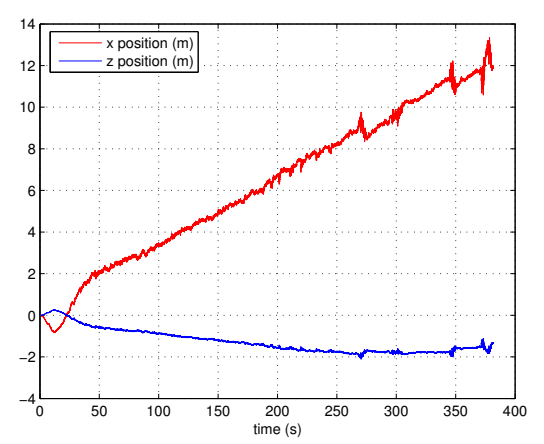

Fig. 5. When no magnetic disturbances are present, the position estimates (slowly) diverge over time.
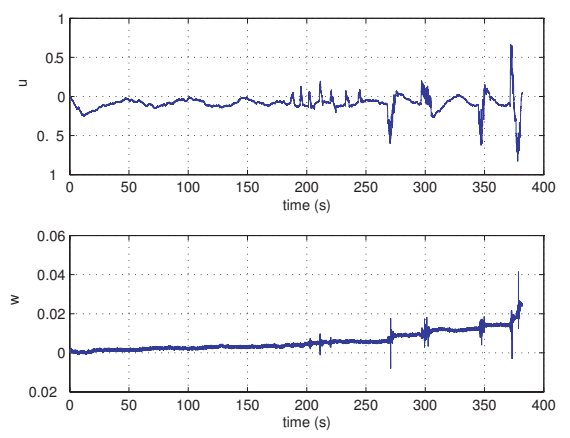

Fig. 6. When no magnetic disturbances are present, the velocities estimates do not remain close to zero. This is a major problem for position estimation.

\section{Experimental results}

For each conducted experiment, we present speed and position estimates histories. The $\mathrm{z}$-axis of our sensor is pointing down, perpendicular to the rail, the $\mathrm{x}$-axis is aligned with the rail. (blue plots refer to the z-axis, red ones refer to the $\mathrm{x}$-axis). A unique set of tuning parameters is used throughout the tests. We give a position reference in black.

1) Outdoor experiment: We choose a non magnetic perturbed area. Due to the lack of magnetic disturbances there is no significant improvement over a classic IMU Kalman filter. Figure 5 and Figure 6 present the obtained results. The position errors diverge over time. Without magnetic disturbances, the filter can not get rid of errors in velocities. The situation is very different indoors, as will be demonstrated next. Interestingly, it can be noted that magnetic perturbations bring sufficient information when the experiment is located within a $5 \mathrm{~m}$ disc around a light-pole.

2) Indoor horizontal experiment: First, a short distance displacement is performed. As can be seen in Figure 7, the (non overshooting) ramps transients are well estimated as long as they are reasonably fast. When the transients are too quick, position bias and overshoots appear. The actual accelerations are in fact way out of the range of expected values (which standard deviation was defined as $0.5 \mathrm{~m} . \mathrm{s}^{-2}$ ). In both cases, the velocities are well estimated. Asymptotically, these estimates remain close to zero, which could not be achieved

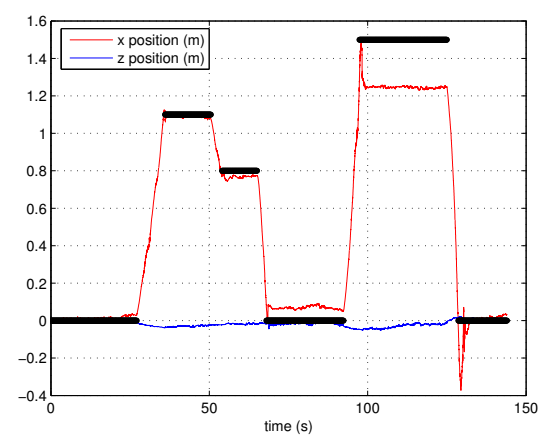

Fig. 7. Short range displacements. Position along the horizontal rail estimate is reported. A bias appears when the transients become too fast.
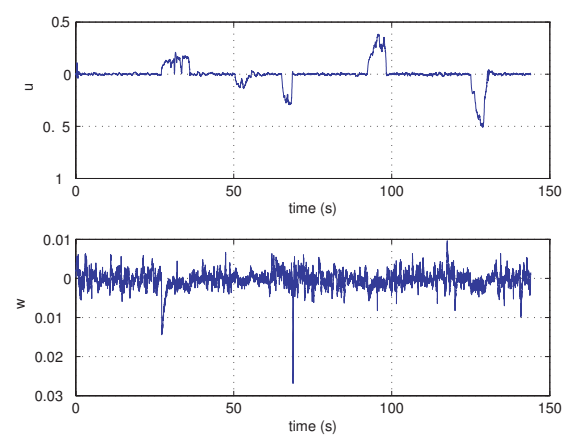

Fig. 8. Short range displacements. Velocities estimates are reported. At rest, they remain close to zero.

without taking advantage of the magnetic disturbances (one can compare Figure 8 and Figure 6). Precise measurements on our experiment reveal that the position error is below $5 \mathrm{~cm}$ over $150 \mathrm{~s}$ of tests.

3) Indoor long time horizontal experiment: A standard benchmark for IMU consists in measuring long-term errors during quasi-static transients. Such an experiment stresses the quality of low-frequency noises rejection. It is critical because of the already discussed integration process. We performed a 30 minutes experiment corresponding to a $2 \mathrm{~m}$ displacement. Results are summarized in Figure 9. Noticeably, the observed $90 \mathrm{~cm}$ position error is very low. This experiment was also used to derive the magnetic field gradient estimates presented in Figure 4. Velocities estimates reported in Figure 10 have a not surprisingly low mean value of $3 e-5 \mathrm{~ms}^{-1}$.

4) Indoor 15 deg experiment: The experimental test bed was oriented to be pointing down with an angle of $15 \mathrm{deg}$. Thanks to this, we could also get information from the gradient of the magnetic field with respect to the $z$-direction. Progressively, we moved the IMU forward by $1.8 \mathrm{~m}$ (two $30 \mathrm{~cm}$ steps followed by three $15 \mathrm{~cm}$ steps, a $30 \mathrm{~cm}$ step and eventually one $15 \mathrm{~cm}$ step and one $30 \mathrm{~cm}$ step). Then we moved it back at the starting point and performed two large steps ( $1.5 \mathrm{~m}$ forward and back). One can easily recognize this motion in Figure 11. Again velocities, reported in Figure 12, 


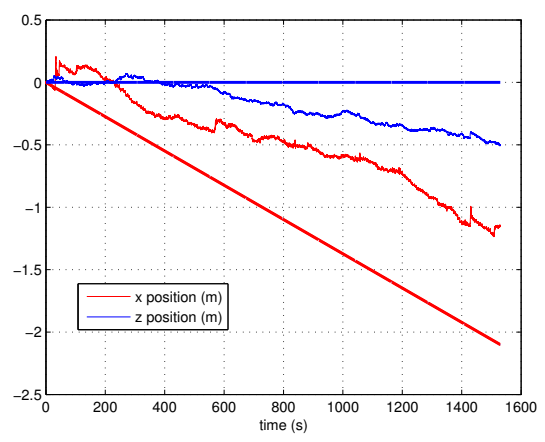

Fig. 9. Long-term low distance displacement. Reference positions are straight lines. Position estimates feature a low long-term error.
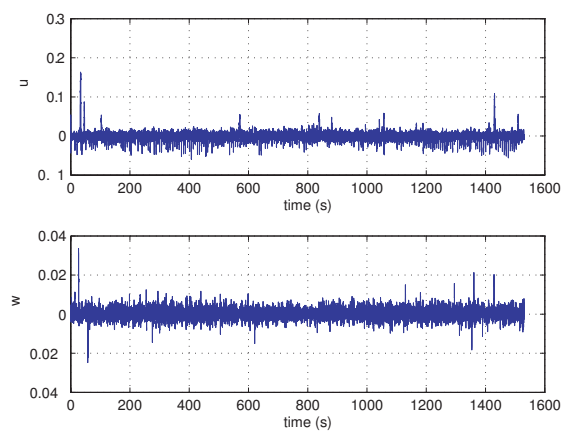

Fig. 10. Long-term low distance displacement. Velocities estimates.

remain close to zero when the IMU is at rest.

\section{CONCLUSIONS AND FUTURE DIRECTIONS}

On our current simple testbed, we obtained some preliminary results that stress the relevance of using magnetic disturbances to improve the position estimation accuracy of IMU signals filtering. We are currently building a more advanced setup for extensive three dimensional testing. Instrumentation, especially position and velocities measurements, is a key issue that remain to be addressed in this 3D configuration. On the performance improvement side, we believe there is room for refinements in the sensors modeling using higher order filters. It can be also expected that different filtering techniques can be used, since our approach merely relies on physics (Maxwell's equation). At last, we would like to mention that we plan to use these position estimators for the control of mobile wheeled robots that are asked to move both indoor and outdoor.

\section{REFERENCES}

[1] R. Abraham, J. E. Marsden, and T. Ratiu. Manifolds, Tensor Analysis, and Applications. Springer, second edition, 1988.

[2] P. Castillo, A. Dzul, and R. Lozano. Real-time stabilization and tracking of a four rotor mini rotorcraft. IEEE Trans. Control Systems Technology, 12(4):510-516, 2004.

[3] S. Changey, D. Beauvois, and V. Fleck. A mixed extended-unscented filter for attitude estimation with magnetometer sensor. In Proc. of the 2006 American Control Conference, 2006.

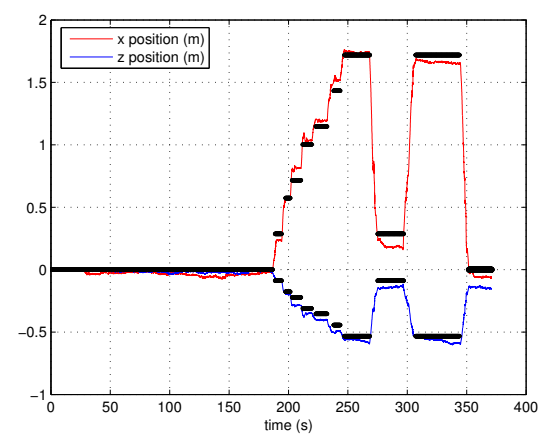

Fig. 11. Succession of steps when the experimental testbed is pointing down with a 15 deg angle. Position estimates.
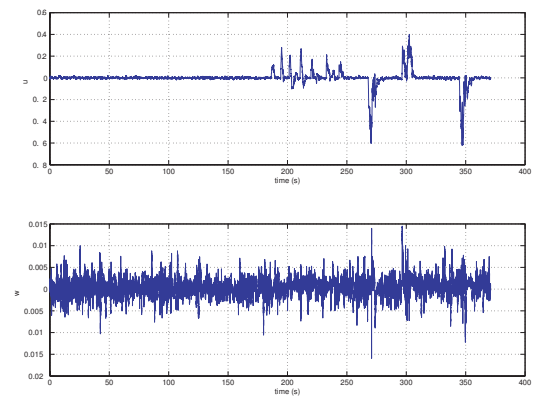

Fig. 12. Succession of steps when the experimental testbed is pointing down with a 15 deg angle. Velocities estimates.

[4] P. Faurre. Navigation inertielle et filtrage stochastique. Méthodes mathématiques de l'informatique. Dunod, 1971.

[5] M. S. Grewal, L. R. Weill, and A. P. Andrews. Global positioning systems, inertial navigation, and integration. Wiley Inter-science, 2001.

[6] C. Jekeli. Precision free-inertial navigation with gravity compensation by an onboard gradiometer. J. Guidance, Control and Dynamics, 29(3):704-713, 2006.

[7] D. Jung, E. J. Levy, D. Zhou, R. Fink, J. Moshe, A. Earl, and P. Tsiotras. Design and development of a low-cost test-bed for undergraduate education in UAVs. In Proc. of the 44th IEEE Conf. on Decision and Control, and the European Control Conference 2005, 2005.

[8] S. Kehl, W.-D. Pölsler, and M. Zeitz. Vehicle path-following with a GPS-aided inertial navigation system. In T. Meurer, K. Graichen, and E.D. Gilles, editors, Control and Observer Design for Nonlinear Finite- and Infinite-Dimensional Systems, LNCIS, pages 285-300. Springer, Berlin, 2005.

[9] R. Mahony, T. Hamel, and J.-M. Pflimlin. Complementary filter design on the special orthogonal group SO(3). In Proc. of the 44th IEEE Conf. on Decision and Control, and the European Control Conference 2005, 2005.

[10] Department of Defense. Network centric warfare. Technical report, Report to Congress, 2001.

[11] R. Sabri, C. Putot, F. Biolley, C. Le Cunff, Y. Creff, and J. Lévine. Automatic control methods for positioning the lower end of a filiform structure, notably an oil pipe, at sea. U.S. Patent 7,066,686, Institut Francais du Pétrole, 2006.

[12] Y. Yang and J.A. Farrell. Magnetometer and differential carrier phase GPS-aided INS for advanced vehicle control. IEEE Trans. Robotics Automation, 19(2):269-282, 2003.

[13] H. Zhao and R. Shibasaki. A vehicle-borne urban 3-d acquisition system using single-row laser range scanners. IEEE Trans. on Systems, Man and Cybernetics, Part B, 33(4):658-666, 2003. 